\title{
LOS DDHH DE LAS MUJERES DESDE UNA PERSPECTIVA DE GÉNERO CON UN ENFOQUE INVESTIGATIVO INNOVADOR
}

\author{
Marco Luis Fernández Leal ${ }^{1}$
}

DOI: https://doi.org/10.5377//rd.v42i1.12938

\section{RESUMEN:}

El presente artículo investigativo en materia de DDHH de las mujeres, tuvo como propósito general: analizar la importancia de los DDHH de las mujeres desde una perspectiva de género con un enfoque investigativo innovador, como propósitos específicos: Develar los DDHH de las mujeres, analizar el concepto de Derechos Humanos y derechos Humanos de las Mujeres. Identificar los Instrumentos internacionales en materia de Derechos Humanos de las mujeres con perspectiva de género. Comprender el contexto de los DDHH de las mujeres desde una perspectiva de género con un enfoque investigativo innovador, desde las voces de los actores sociales. Se abordó bajo el paradigma interpretativo, enfoque cualitativo, orientado en la fenomenología, que interpreta y comprende los significados en los discursos de las personas. La técnica para la recolección de información fue la entrevista en profundidad y validación de expertos, dirigida a tres (03) profesionales, Dos (02) Abogados, un (01) Criminólogo. Los hallazgos obtenidos develaron que los DDHH de las mujeres deben ser investigados con perspectiva de género, reconocer la mujer como sujeto de derecho, resaltar los instrumentos internacionales en pro de prevenir, erradicar y sancionar cualquier tipo de discriminación, violencia que atente contra ella, con un enfoque investigativo innovador.

PALABRAS CLAVES: DDHH de las Mujeres, Perspectiva de Género, Enfoque investigativo innovador.

Fecha de recepción: 31/8/21

Fecha de aprobación: 1/11/2021

1 Abogado por la Universidad de Los Andes ULA/Venezuela, Diplomado en Ciencias Forense ULA, Magister en Ciencias Penales y Criminológicas, Docente de la cátedra de Delito Contra Las Persona, Universidad Yacambú UNY/Venezuela.

Correo Electronico: abg.fernandezmarco@gmail.com 


\title{
WOMEN'S HR FROM A GENDER PERSPECTIVE WITH AN INNOVATIVE RESEARCH APPROACH
}

\author{
Marco Luis Fernández Leal
}

DOI: https://doi.org/10.5377//rd.v42i1.12938

\begin{abstract}
:
The present investigative article on women's human rights had the general purpose: to analyze the importance of women's human rights from a gender perspective with an innovative investigative approach, as specific purposes: To reveal the human rights of women, to analyze the concept of Human Rights and Human Rights of Women. Identify international instruments on the Human Rights of women with a gender perspective. Understand the context of women's human rights from a gender perspective with an innovative investigative approach, from the voices of social actors. It was approached under the interpretive paradigm, a qualitative approach, oriented in phenomenology, which interprets and understands the meanings in people's discourses. The technique for the collection of information was the in-depth interview and validation of experts, directed to three (03) professionals, Two (02) Lawyers, one (01) Criminologist. The findings obtained revealed that the human rights of women should be investigated with a gender perspective, recognize women as a subject of law, highlight the international instruments in favor of preventing, eradicating and punishing any type of discrimination, violence that attempts against her, with an innovative research approach.
\end{abstract}

KEY WORDS: Human Rights of Women, Gender Perspective, Innovative Investigative Approach.

Date received: 8/31/21

Approval Date: 11/1/2021

2 Lawyer from the Universidad de Los Andes ULA / Venezuela, Diploma in Forensic Sciences ULA, Magister in Criminal and Criminological Sciences, Professor of Crime Against Persons, Universidad Yacambú UNY / Venezuela, Email: abg.fernandezmarco@gmail.comccc abg.fernandezmarco@gmail.com 


\section{INTRODUCCIÓN}

Esta investigación está enfocada en generar ideas innovadoras, aportar elementos fundamentales y esenciales a la administración de justicia, nacional e internacional, así como a la sociedad académica investigativa jurídica y las ciencias penales y criminológicas en todos sus niveles, no es el hecho de copiar y pegar un texto de un autor, es de contribuir, de producir y proyectar saberes dogmáticos jurídicos de una forma sistemática y hermenéuticamente adecuada, ajustada a la realidad investigativa, resaltando la esencia investigativa, considerando a la mujer como un sujeto de derecho y no como un objeto dentro de un proceso investigativo, en el marco de los instrumentos legales internacionales en materia de derechos humanos con enfoque de género.

La desigualdad entre el hombre y la mujer, ha estado presente desde muchos antes de la declaración universal de los Derechos Humanos (1948) y por tal razón, es importante referir, que el género más vulnerado en todo sus aspectos y en su extrema violación de Derechos Humanos ha sido la mujer, por esta razón, el dominio patriarcal y machista que ha venido ejecutando el hombre hacia la mujer, ha sido constante y reiterado en el tiempo, y de considerarla como un objeto y no como un sujeto de derecho, claro está, que desde el tiempo de Roma, dentro del Derecho Romano, se consideraba a la mujer en segundo plano por no ostentar los tres estatus que se necesitaban para el momento, mientras que el hombre, era considerado como el paterfamilias, es decir, era quien ejercía la autoridad dentro del núcleo familiar y social mientras que la mujer se limitaba a sus deberes del hogar.

ISSN: 2521-5159 (En Línea)
También, es importante resaltar, que la mujer, en virtud de una costumbre social común en el Mundo Antiguo, sus facultades estaban restringidas al máximo: se les negaba el ius suffragii y el ius honorum, de forma que no podían votar y mucho menos postularse para un cargo público. Castan (2020), la discriminación era infalible por el simple hecho de ser mujer, automaticamente la mujer ya tenía un rol definido y limitado. los derechos que le correspondía a una mujer por el simple hecho de ser persona era sometido a consentimiento del hombre bajo un dominio y una subordinación machista imperial. Por tanto, Los romanos justificaban el sometimiento y la violencia que generaban en ellas, argumentando que la mujer sufría de fragilidad de ánimo y carecía de derechos, por lo que necesitaba estar bajo la tutela masculina.

Es inevitable, pasar por el tiempo de Roma, o el Derecho Romano, sin recordar esas clases de Derecho Romano, donde los mismo profesores nos hacían hincapié que la mujer era considerada en esa época como un objeto, y que la misma debía limitarse a realizar muchas cosa, hoy en día, por elevar una investigación no en contra del hombre sino más bien determinando la importancia que tiene la intervención de investigar con esencia de mujer y perspectiva de género los hechos que agrede la integridad, la moral y la estabilidad emocional y psicológica de las mujeres por el simple hecho de ser la esencia indispensable del hombre.

Los Derechos Humanos han ido evolucionado a medida que los años pasan, se hace presente investigaciones que determinan el grado de vulnerabilidad que actualmente se vive, y más en el mundo del género femenino, las muertes brutales y misóginas de mujeres por su condición de género tal cual como lo refiere Fundación Ayuda en Acción (2020):

Revista de Derecho. Vol. 42, No. 1, Año 2021|-139 
En Bolivia: en un país donde en 2015 el 10\% de las víctimas mortales de la violencia de género eran menores de 18 años, 83 mujeres fueron asesinadas entre enero y agosto de 2020. Durante la cuarentena hubo 53 casos. Colombia: según el Observatorio de Feminicidios en Colombia, 445 mujeres fueron asesinadas hasta septiembre. Durante la cuarentena, se alcanzó la cifra de 243 feminicidios. Respecto a Costa Rica: de enero a septiembre de 2020 se han registrado 10 feminicidios íntimos en el país. En el mismo orden, Ecuador: existe un informe elaborado por varias organizaciones dentro de la plataforma Alianza Mapeo donde identifican 748 mujeres asesinadas desde 2014 hasta marzo de 2020. Desde el 1 de enero al 2 de marzo de 2020 se contabilizaron 17 feminicidios.

A lo que corresponde, El Salvador: el Observatorio de Violencia de Ormusa registra 71 feminicidios hasta el 13 de agosto de 2020. La cifra ha sufrido una bajada considerable con respecto a las del año anterior. mientras que Guatemala: según cifras conocidas en octubre de 2020, el Observatorio de la Mujer, del Ministerio Público, reconocía 319 feminicidios. Por su parte, Honduras: hasta el 30 de septiembre se registraron 195 feminicidios en el país. Desde el establecimiento del toque de queda por la COVID-19 se han registrado 126 muertes violentas de mujeres.

Por tanto, México: en 2020 el Observatorio de Feminicidios de México ha reportado 724 feminicidios hasta el mes de septiembre. Nicaragua: en los primeros 8 meses de 2020 el Observatorio Católicas por el Derecho a Decidir registró 50 feminicidios. a lo que respecta Paraguay: hasta agosto se registraron 20 feminicidios en el país sudamericano. Perú: la Defensoría del Pueblo reconoce hasta octubre 100 casos de feminicidios reconocidos oficialmente y otras 45 muertes violentas de mujeres que están en estudio. Y por último se tiene a Venezuela: 172 mujeres fueron asesinadas por violencia de género en los primeros ocho meses del año.

Estas cifras, nos revelan el grado de vulneración de Derechos Humanos que viven las mujeres de modo reiterado, sin embargo, muchos paises de latinoamerica se acogen a los convenios y tratados internacionales en materia de protección de los Derechos Humanos de las mujeres, a objeto de trabajos no solo en la sanción de estos hechos violentos, sino también, de trabajar en función de la prevención en manos de las ciencias penales interdisciplinarias como las ciencias penales y criminológicas.

En su defecto la Organización Mundial de la Salud (2021) Advierte que las mujeres jóvenes se encuentran entre las que más riesgo corren, Los nuevos datos hechos públicos por la Organización Mundial de la Salud (OMS) y sus asociados demuestran que la violencia contra la mujer continúa siendo un problema generalizado y devastador y que se empieza a sufrir a edades alarmantemente tempranas. Cerca de 736 millones de mujeres (es decir, una de cada tres) sufren violencia física o sexual infligida por un compañero íntimo o agresiones sexuales perpetradas por otras personas, unas cifras que se han mantenido estables a lo largo del decenio más reciente.

A su vez, dicha Organización que vela por los DDHH de las mujeres, ratifica que esta violencia empieza temprano: una de cada cuatro mujeres de entre 15 y 24 años que han mantenido alguna relación íntima habrán sido objeto de las conductas violentas de un compañero íntimo cuando cumplan 25 años. Los aportes extraídos 
de dichas referencias documentales, permiten visualizar en modo concreto que no hay ejecución o aplicación de los instrumentos internacionales en materia de derechos humanos en beneficio de la mujer a pesar de que es una responsabilidad que tienen todos los países de Latinoamérica que son parte y han ratificado convenios y tratados internacionales en protección de los derechos de las mujeres.

La Problemática Investigativa de este tema se enfoca en dejar claro que los hechos de violación de Derechos Humanos de las mujeres son constantes y reiterados en el tiempo, son cifras altas que se reflejan y que no hay un buen uso o aplicación de estos instrumentos legales, la impunidad a evadido y se ha convertido en un cáncer en estado de metástasis, la subordinación del hombre hacia la mujer no tiene limite, ellos ejecutan su conducta en cualquier espacio de la vida tanto públicos como privados, no importa la edad de la mujer, dentro de este contexto entran niñas, adolescente y mujeres adultas mayores.

La Justificación investigativa esta direcciona en producir un conjunto de definiciones epistemológicas que aborden el contexto investigativo, generando conocimientos solidos basado en la innovación, con esencia de mujeres y perspectiva de género, aportando así elementos solidos a los sistemas de justicia penal, instituciones públicas/privada, ONG dedicada en brindar protección al sector vulnerado y violentado de sexo femenino por su condición de género, si menoscabar los derechos humanos de los hombre, respetar el derecho de igualdad y equidad, y aun más, de que la sociedad investigativa académica y docente tome conciencia a la hora de investigar hechos donde se vulneren los derechos humanos de las mujeres.
Por tanto, para tener una óptica más clara al respecto está articulo investigativo e innovador, con perspectiva de género, se considerará una metodología bajo el paradigma interpretativo, enfoque cualitativo, orientado en la fenomenología, que interpreta, comprende los significados en los discursos de las personas, para darle el enfoque innovador y a partir de la técnica de entrevista obtener por parte de los tres (03) especialista en materia de derechos humanos, constituido por dos (02) Abogados y un (01) Criminólogo. por razón de resaltar la importancia Los DDHH de las mujeres desde una perspectiva de género con un enfoque investigativo innovador.

Esta temática, es un aporte importante para futuras investigaciones, ya que existen escasas aportaciones conceptuales y empíricas en bibliografías que abordan el tema, con un sentido de pertenencia distinto, a las acostumbradas a leer, es por esta razón que se investiga con esencia de mujer y perspectiva de género, es decir, valorar a la mujer, respetar sus derechos humanos sin menoscabar al de los hombres y considerarla como un sujeto de derecho.

\section{FUNDAMENTO EPISTEMOLÓGICO}

Los fundamentos epistemológicos que sustenta esta producción investigativa son los siguientes:

\subsection{Concepción de la definición de DDHH y DDHH de las Mujeres}

Los Derechos Humanos, es aquella herencia que ostenta el ser humano al momento de la concepción, es esa esencia única que nos permite desenvolvernos libremente, en pro de la igualdad, equidad, el respeto con los otros. en este espacio investigativo e innovador, la idea no es repetir sino generar ideas, la historia de los DDHH ya están 
prescritos y determinado en normativas, este espacio, busca es poder concebir una definición ajustada entendible y comprensible, por tanto, es reflejar las siguientes definiciones:

Mertus, Butegwa, Thomas y Schuler (2000) refiere que, los derechos humanos son aquellos que todo ser humano posee y que tiene el derecho de disfrutar, simplemente por su condición de ser humano. pág. 10, esta es una definición en sentido general, por así considerarla que establece elementos esenciales que determina la condición de una persona con relación a los derechos que les corresponde y que son inherente para el mismo.

Por otro lado, La Comisión Nacional de Los Derechos Humanos (2021) considera que "Los Derechos Humanos son el conjunto de prerrogativas sustentadas en la dignidad humana, cuya realización efectiva resulta indispensable para el desarrollo integral de la persona. Este conjunto de prerrogativas se encuentra establecido dentro del orden jurídico nacional, en nuestra Constitución Política, tratados internacionales y las leyes". Aunado a eso la comisión refiere qué, Los derechos humanos son derechos inherentes a todos los seres humanos, sin distinción alguna de nacionalidad, lugar de residencia, sexo, origen nacional o étnico, color, religión, lengua, o cualquier otra condición. Todos tenemos los mismos derechos humanos, sin discriminación alguna. Estos derechos son interrelacionados, interdependientes e indivisibles.

El aporte que hace la CNDH, es fundamental, en razón de que dicha institución se direcciona a la protección de los derechos humanos resaltando la característica esencial de estos derechos, como derechos progresivos irrenunciable y universales, un ente dedicado a la protección de los iguales ante las leyes, convenios y tratados internacionales en materia de derechos humanos, sin discriminar y sin considerar la preferencia de derechos entre los hombres y mujeres. Por tanto, estas palabras son ratificadas por la misma comisión al referir, que los derechos humanos universales están a menudo contemplados en la ley y garantizados por ella, a través de los tratados, el derecho internacional consuetudinario, los principios generales y otras fuentes del derecho internacional.

Siguiendo el mismo orden de idea, es de imperiosa necesidad resaltar los principios que contextualiza la $\mathrm{CNDH}$, los cuales son los siguientes;

- El principio de la universalidad. Todas las personas son titulares de todos los derechos humanos. Dicho principio se encuentra estrechamente relacionado a la igualdad y no discriminación. No obstante, para lograr la igualdad real se debe atender a las circunstancias o necesidades específicas de las personas.

- Principio de Interdependencia: Consiste en que cada uno de los derechos humanos se encuentran ligados unos a otros, de tal manera que el reconocimiento de uno de ellos, así como su ejercicio, implica necesariamente que se respeten y protejan múltiples derechos que se encuentran vinculados.

- Principio de Indivisibilidad: Implica que los derechos humanos no pueden ser fragmentados sea cual fuere su naturaleza. Cada uno de ellos conforma una totalidad, de tal forma que se deben reconocer, proteger y garantizar de forma integral por todas las autoridades. 
- Principio de interdependencia e indivisibilidad: Todos los derechos humanos, sean éstos los derechos civiles y políticos, como el derecho a la vida, la igualdad ante la ley y la libertad de expresión; los derechos económicos, sociales y culturales, como el derecho al trabajo, la seguridad social y la educación; o los derechos colectivos, como los derechos al desarrollo y la libre determinación, todos son derechos indivisibles, interrelacionados e interdependientes. El avance de uno facilita el avance de los demás. De la misma manera, la privación de un derecho afecta negativamente a los demás.

- Principio de Progresividad: Constituye una obligación del Estado para asegurar el progreso en el desarrollo constructivo de los derechos humanos, al mismo tiempo, implica una prohibición para el Estado respecto a cualquier retroceso de los derechos. El Estado debe proveer las condiciones óptimas de disfrute de los derechos y no disminuir ese nivel logrado.

Es importante y determinante determinar la importancia de dichos principios que se perfecciona a medida que se van ejecutando dichos derechos que nos corresponde a todos por iguales, derechos que nacen con nosotros $\mathrm{y}$ que mueren con nosotros y sin embargo hasta después de muertos se debe hacer justicia por nuestra muerte o las muertes violentas y dudosas de aquellas mujeres y hombres que hasta ahora han quedado impune.

Una vez contextualizada la definición epistemológica de derechos humanos, se hace más eficaz para el desarrollo de la definición de los derechos humanos de las mujeres.
Los derechos humanos de las mujeres son los mismo que los del hombre con las misma características y principios sin exclusión y sin discriminación. los derechos humanos que le pertenecen a las mujeres nacen con ellas misma, el problema es que hace siglos un conjunto de grupos de hombres machista no consideraban que la mujer podría ser tan grande o igual que ellos, pero realmente hasta ahora esa conducta visceral del hombre hacia la mujer no ha cambiado. sin embargo, en esta investigación se busca reflejar el aspecto innovador e investigativo de hacer visible la independencia de la mujer frente al hombre, que, en estos tiempos se encuentra en igualdad, en todos los aspectos, laboral, económico, jurídico, político, profesional y académico investigativo.

Los derechos humanos de las mujeres son un conjunto de derechos y deberes que tienen las mujeres en igualdad que los derechos de los hombres, solo es de comprender y dejar claro, de que como existe grado de igualdad y equidad entre ambos, el hombre no debe de aprovecharse de este concepto tan efímero. Esto, es en el aspecto jurídico legal.

Los DDHH de las Mujeres, han pasado por un proceso inestable, el proceso de reconocimiento como sujeto de derecho dentro de la sociedad machista fue y ha sido un proceso inaceptado, en virtud de que estos derechos hasta ahora se siguen vulnerando, la igualdad y la equidad, ha sido la lucha permanente por las organizaciones activista, de que se haga cumplir el espíritu del legislador, y que los hechos violentos donde la víctima sea la mujer sean investigados con perspectiva de género. Es tanto así, que es importante referir lo que considera Camacho (2016) El solo hecho de estar formalizados en un instrumento jurídico de tan alto rango, no los convierte en Derechos Humanos. Sin los movimientos sociales que los 
hagan respetar y sin las transformaciones que los incorpore a la cultura, no son, no existen, no constituyen Derechos Humanos.

Lo antes expuesto por dicho autor, refleja taxativamente que no es tanto el reconocimiento de dichos derechos es de que se cumpla su voluntad, que se aplique salvaguardando los derechos de las mujeres, y ejecutándose dentro del marco normativo de la igualdad y equidad de género.

\subsection{La Mujer Sui Iuris con genus perspective}

Es importante referir que la mujer es considerada por un gran porcentaje de hombre como la mujer de la casa, y que la misma debe hacer los deberes del hogar, que no puede ir un paso adelante de ellos, y que todo lo que ellas hacen debe ser bajo la supervisión de ellos, este es un emblema que hasta ahora se mantiene, y esto se traduce en que la mujer es lo que el hombre quiere que sea, no lo que ella quiere ser, es un objeto, un trofeo, la esclava del hogar.

La mujer es un sujeto de derecho, un ser humano, una persona de carne y hueso, con sentimientos, emociones, que siente y padece, y que busca lograr sus sueños, ser feliz, crecer y proyectarse hacia el futuro. la mujer es la esencia única que no debe ser tocada ni con el pétalo de una rosa. la mujer a través del tiempo y por medio de los movimientos feminista y la declaración de los derechos universales del hombre y la mujer porque asi deben llamarse para respetar la perspectiva de género, la igualdad del ser humano ante el estado y la ley. Fernández (2021) refiere en su investigación, que la mujer debe ser vista como un sujeto de derecho y no como un objeto. en virtud de resaltar y dejar claro la postura de la mujer frente al hombre en pro de la igualdad entre ambos seres humanos.
Los derechos Humanos de las Mujeres con una Perspectiva de Género $\mathrm{y}$ un enfoque investigativo innovador El estudio sobre los derechos humanos de las mujeres ha conllevado a muchos investigadores en explorar la esencia de la misma, no es de escribir lo que mucho han escrito, es de generar un aporte investigativo con una esencia única, eficaz entendible y comprensible, la historias de los derechos humanos en general y de los derechos humanos de las mujeres la conocemos, pero lo que se busca en este tema investigativo lo cuán es importante el uso de la perspectiva de género con el fin de lograr ese enfoque que lo hace único de otras investigaciones y que a su vez le deja un gran aporte a la comunidad científica investigativa en todo sus niveles.

Por tanto, en esta investigación se tomará en cuenta que la "Perspectiva de Género" se considera como la forma o el método idóneo, de cómo se debe investigar hechos donde se encuentre involucrada una mujer, respetando los derechos humanos de ambas partes, resaltando la igualdad y equidad, de que la mujer sea vista como un sujeto de derecho pleno en todo su contexto, al objeto de evitar la impunidad, la victimización y lograr el acceso a la justicia. Fernández (2021). Este aporte refleja la calidad y la esencia metodológica innovadora, en pro de los derechos humanos en resguardar y respetar los derechos humanos de los hombres y las mujeres, con perspectiva de género.

La óptica de aportar y de iniciar una investigación donde no exista la exclusión del ser humano, es decir discriminación del hombre hacia la mujer y viceversa, es un enfoque innovador en el mundo académico investigativo en todos sus niveles. Pese, esta investigación la aplicación de la perspectiva de género con enfoque investigativo 
innovador, en mejorar la información académica sobre los derechos humanos de las mujeres.

\subsection{La Perspectiva de Género en los Instrumentos Legales Internacionales sobre los DDHH de las Mujeres}

Promover la Protección y la seguridad jurídica y social a todas las mujeres por su esencia única es el objetivo en que se enfoca este tema innovador, desentrañar esos aspectos con perspectiva de género que subyace de un conjunto de normas internacionales que son adaptado por muchos países en su norma suprema denominada "Constitución" y que la misma norma lo considera derechos internacionales constitucionales o también es considerado como los derechos de los derechos humanos. Por tanto, se realizará en este espacio un breve recorrido de esas normas que revisten la esencia de mujer con perspectiva de género a objeto de poder comprender este aspecto epistemológico.

Es inevitable empezar hacer referencia a lo que se desentraña de la Carta de las Naciones Unidas de 1945: Al reafirmar la fe en los derechos fundamentales del hombre, en la dignidad y el valor de la persona humana, en la igualdad de derechos de hombres y mujeres... concibe la esencia de perspectiva de género al reflejar el derecho a la igualdad y equidad, en generalizar y no particularizar. Aunado a eso se precisa un pequeño extracto de lo que refieren los artículos 1 , 22 y 23 de la Declaración la Declaración Universal de los Derechos Humanos en 1948, se refieren expresamente a la dignidad humana: "Todos los seres humanos nacen libres e iguales en dignidad y derechos y dotados como están de razón y conciencia deben comportarse fraternalmente los unos con los otros" (artículo 10.). 13. El Pacto Internacional de Derechos Civiles $\mathrm{y}$
Políticos de Naciones Unidas de 1966 reitera las mismas ideas en su preámbulo: “...Reconociendo que estos derechos se derivan de la dignidad inherente a la persona humana...", conceptos que se vuelven a manifestar, como es natural, en el Pacto Internacional de Derechos Económicos, Sociales y Culturales de ese mismo año.

Respetando el mismo orden de idea, se resaltan aquellos instrumentos internacionales que desentraña la perspectiva de género, no a favor de ningún género en específico, sino de afianzar la igualdad, la equidad de género, resaltando los derechos vulnerados que en esta temática son los derechos humanos de las mujeres. son los siguientes instrumentos legales internacionales:

El Comité para la Eliminación de todas las formas de Discriminación de la Mujer, indica en su artículo 1, lo siguiente: "La violencia contra la mujer es una forma de discriminación" dicha norma a su vez, guarda relación, con la Recomendación General No.19, en la que se señala que la violencia contra la mujer contraviene la Convención sobre la Eliminación de todas las Formas de Discriminación a la Mujer" (CEDAW) porque esta violencia "se dirige a ella porque es mujer o la afecta en forma desproporcionada". Recomienda que:

a. Los Estados Parte adopten medidas apropiadas y eficaces para combatir los actos públicos o privados de violencia por razones de sexo.

b. Los Estados Parte velen por que las leyes contra la violencia y los malos tratos en la familia, la violación, los ataques sexuales y otro tipo de violencia contra la mujer protejan de manera adecuada a todas las mujeres y respeten su integridad y su dignidad. Debe 
proporcionarse a las víctimas protección y apoyo apropiados. Es indispensable que se capacite a los funcionarios judiciales, los agentes del orden y otros funcionarios públicos para que apliquen la Convención.

A su vez, La Declaración para la Eliminación de la Violencia Contra la Mujer emanada de la Conferencia Mundial de los Derechos Humanos realizada en Viena (1993), reconoce que la violencia basada en género “... constituye una manifestación de relaciones de poder históricamente desiguales entre el hombre y la mujer, que han conducido a la dominación de la mujer y a la discriminación en su contra por parte del hombre eimpedidoeladelanto plenodela mujer, (...) respecto a la Convención Interamericana para Prevenir, Sancionar y Erradicar la Violencia contra la Mujer (Convención de Belem do Para) es el primer instrumento vinculante de derechos humanos destinado a este fenómeno (adoptada en 1994). En ella se dispone que "Toda mujer tiene derecho a una vida libre de violencia..."

En mismo orden de idea, el Estatuto de Roma de la Corte Penal Internacional Es otro instrumento de derechos humanos de especial significación ante los femicidios/ feminicidios de mujeres, en tanto es competente para atribuir responsabilidad penal internacional a individuos por la comisión de delitos de genocidio, lesa humanidad y guerra. La violencia contra las mujeres por parte del Estado y sus agentes, evitar la impunidad y prevenir que los hechos de violencia de se sinteticen en la sociedad. Por tanto, el Estatuto de la Corte Penal Internacional incluye disposiciones claras en relación con la perspectiva de género como por ejemplo la obligación de investigar todo hecho de violencia de género y la prohibición de hacer distinciones basadas en género que tengan efectos adversos en hombres o mujeres.

Es de gran importancia considerar en este espacio investigativo que todo convenio y tratados internacional que verse sobre las materias de derechos humanos de las mujeres, y que afecte la integridad, la moral, la libertad y la vida de misma es un aporte innovador con esencia investigativa que sirve de aporte a futuros investigadores.

La razón de hablar de la importancia de los Instrumentos legales internacionales en materia de derechos humanos y aún más los derechos humanos de las mujeres son por razones que establece -Mertus, Butegwa, Thomas y Schuler(2000):

- Garantizan a los individuos derechos específicos. - Establecen obligaciones para los Estados. - Crean mecanismos para supervisar el cumplimiento de obligaciones por parte de los Estados y permiten que los individuos soliciten reparaciones por las violaciones de sus derechos Pág.13. En virtud de que cada una de las bases legales ante referidas reflejan la perspectiva de género con enfoque innovador, intrínsecamente son normas que son direccionadas en explorar y hacer valer la realidad de los derechos de las mujeres en todo su espacio y contexto social.

\section{METODOLOGÍA}

El aspecto metodológico de esta investigación con perspectiva de género con un enfoque innovador se direccionó bajo el Paradigma Cualitativo y tiene como propósito develar el sentido y significado que le asignan los actores sociales a los aspectos resaltantes en relación con el tema investigativo. Su estructura dinámica tal como lo expresan Taylor y Bogdan (1987), quienes 
indican que la investigación cualitativa "es aquella que produce datos descriptivos con las propias palabras de las personas, habladas o escritas y la conducta observable" ( $\mathrm{p} 20$ ). mediante el método fenomenológico, en tal sentido, Martínez M (2001) expresa que éste "consiste en el estudio tal como son experimentados, vividos y percibidos por el hombre", permite el abordaje de las realidades desde el marco de referencia interno del sujeto que la vive y experimenta.

En el mismo proceso metodológico se consideró Actores sociales; personas que tienen conexión directa con el tópico investigativo innovador, fueron seleccionados de forma intencional tres (3) actores sociales todos especializados, considerados así, por poseer información que aportan conocimiento de gran importancia para este artículo, por tal razón se distribuyó de la siguiente manera: Dos (2) Abogados (ASA-1, ASA-2); Un (1) Criminólogo (ASC). Se realizó la técnica entrevista a profundidad que sigue el modelo de una conversación entre iguales, mostrando un intercambio formal de preguntas y respuestas, en un proceso que pretende descubrir categorías y establecer coincidencias en la categorización del discurso surgido.

Partiendo de la información obtenida de la entrevista a profundidad, dirigida a los tres (3) actores sociales, se pudo obtener información sólida y sostenibles respecto a este tema investigativo. Por tal razón, se presentará el análisis de los datos producto del estudio realizado, con el propósito de dar respuesta a los propósitos formulados donde se presentará la información obtenida de los tres (03) actores sociales.

\section{Cuadro $\mathrm{N}^{\circ} 1$ Consideración sobre el término DDHH de las Mujeres}

\begin{tabular}{|c|l|}
\hline Actor Social & \multicolumn{1}{|c|}{ Perspectiva } \\
\hline $\boldsymbol{A S A}$-1 & $\begin{array}{l}\text { Son derechos que por hace mucho tiempo } \\
\text { estaba olvidado pero que en la actualidad } \\
\text { están respaldados por un conjunto de } \\
\text { normas internacionales }\end{array}$ \\
\hline $\boldsymbol{A S A - 2}$ & $\begin{array}{l}\text { Son derechos que poseen las mujeres } \\
\text { desde el momento que están en el claustro } \\
\text { materno y que nunca prescriben y se } \\
\text { mantienen en el tiempo. }\end{array}$ \\
\hline $\boldsymbol{A S C}$ & $\begin{array}{l}\text { Son derechos esenciales y fundamentales } \\
\text { de las mujeres y que son universales e } \\
\text { inalienables. . }\end{array}$ \\
\hline
\end{tabular}

De acuerdo con la respuesta aportada por los actores sociales, en resumida cuenta se puede considerar que son apreciable a la esencia investigativa, proyectan elementos, que caracterizan los derechos humanos en sentido general, y en especial a los derechos humanos de las mujeres.

Cuadro $\mathrm{N}^{\circ} \mathbf{2}$ Consideración sobre la perspectiva de género y el enfoque investigativo innovador

\begin{tabular}{|c|l|}
\hline Actor Social & \multicolumn{1}{c|}{ Perspectiva } \\
\hline$A S A-1$ & $\begin{array}{l}\text { La perspectiva de género permite distinguir } \\
\text { a la mujer, resaltar sus aspectos esenciales } \\
\text { sin menoscabar los derechos del hombre, y } \\
\text { el enfoque usado perfecciona el concepto } \\
\text { que se proyecta en el tema investigativo. }\end{array}$ \\
\hline$A S A-2$ & $\begin{array}{l}\text { el uso del metodo de la perspectiva de gene- } \\
\text { ro sumado al enfoque investigativo invador } \\
\text { deja claro que no debe existir desilgualda } \\
\text { ni discriminación en las investigaciones y } \\
\text { que la mujer debe ser vista como un sujeto } \\
\text { de derecho. }\end{array}$ \\
\hline$A S C$ & $\begin{array}{l}\text { este contexto considerado en la investiga- } \\
\text { ción particulariza esta investigación de } \\
\text { otras y le da un sentido de pertenencia úni- } \\
\text { co reforzando el derecho de la igualdad y } \\
\text { equidad de género innovador. }\end{array}$ \\
\hline
\end{tabular}

La perspectiva de los actores respecto a esta pregunta, reflejan con claridad y confiabilidad la innovación que se proyecta en este tema investigativo.

Revista de Derecho. Vol. 42, No. 1, Año 2021 -147 
Cuadro $N^{\circ} 3$ Consideración general de los Actores Sociales respecto al tema Investigativo

\begin{tabular}{|c|l|}
\hline Actor Social & \multicolumn{1}{c|}{ Perspectiva } \\
\hline $\boldsymbol{A S A}$-1 & $\begin{array}{l}\text { Un tema con una perspectiva que proyecta } \\
\text { ideas innovadoras }\end{array}$ \\
\hline $\boldsymbol{A S A - 2}$ & $\begin{array}{l}\text { Es un artículo que sustenta la esencia de } \\
\text { los derechos humanos de las mujeres desde } \\
\text { un punto de vista distinto al acostumbrado } \\
\text { a leer. }\end{array}$ \\
\hline $\boldsymbol{A S C}$ & $\begin{array}{l}\text { un aporte innovador tanto para la justicia } \\
\text { como para las instituciones universitarias } \\
\text { en todos sus niveles. }\end{array}$ \\
\hline
\end{tabular}

La percepción por parte de los actores sociales, concuerdan y enfatizan que dicha investigación está sustentada y es enfocada con ideas innovadoras.

\section{RESULTADOS}

De acuerdo a la información y resultados obtenidos en el proceso de proyección e innovación de ideas y de los fundamentos epistemológicos se puede indicar que se cumplió con los propósitos planteados, entre ellos se pudo considerar que;

La concepción de los aspectos relacionados a los DDHH de las mujeres desde una perspectiva de género con un enfoque investigativo innovador, generaron un enfoque atractivo investigativo e innovador donde se mantuvo la esencia investigativa, se centró en lo fundamental, en aclarar esos aspectos que hacen único a la mujer, así como el proceso de reconocimiento de sus derechos humanos, dentro del ordenamiento jurídico internacional, basado en la igualdad, universalidad y equidad de género. Respecto a la percepción del concepto de Derechos Humanos y derechos Humanos de las Mujeres; permitió determinar claro que la mujer debe ser considerada como un sujeto de derecho y no como un objeto, que sus derechos son iguales que el de los hombres, y que son derechos inalienables.

Otro propósito esencial, es la perspectiva usada en los Instrumentos internacionales en materia de derechos humanos de las mujeres, es de enfatizar que desde la declaración universal de los derechos humanos de las mujeres ya dichas normas concibió la perspectiva de género pero al momento de aplicarla en su mayor parte no lo hacían, por tanto pierden la objetividad, por tal razón que actualmente abunda la impunidad de las muertes violentas de mujeres por su condición de género y un sin fin de violencia que viven la mujeres de una forma constante, resaltando que la perspectiva aparte de ser la esencia innovadora de este artículo es el método que también se pone en marcha para trabajar en pro de la prevención y la igualdad.

Por último, los aportes por parte de los actores sociales del presente artículo investigativo. permitieron centrar este artículo investigativo en un contexto innovador, permitiendo aportar ideas, como de ver a la mujer como un sujeto de derecho derecho y no como un objeto, arropada con derechos fundamentales basado en la igualdad y equidad de género, y de que la misma pueda ser utilizadas por futuros investigadores en su proceso investigativo en materia de derechos humanos de las mujeres con perspectiva de género con enfoque investigativo innovador, con el objeto de evitar la impunidad y tener acceso a la justicia.

\section{DISCUSIÓN}

La proyección intelectual investigativa innovadora de este artículo investigativo con perspectiva de género con un enfoque distinto es por razón de generar un conjunto de 
fundamentos epistemológico que facilite una mejor comprensión relacionados a los derechos humanos de las mujeres. y que otros investigadores tomen con seriedad, respeto y profesionalismo estos temas que general un conjunto de reflexión jurídica dogmática a nivel académico universitario investigativo.

Es de gran importancia referir que las referencia bibliográficas usada en esta investigación fortalecieron aspecto importante, tal como refiere Fernández (2021) la mujer debe ser percibida como un sujeto de derecho, es relevante, procedente e innovador, también, al dar una definición concreta respecto a la Perspectiva de género, los aportes por parte de la CNDH, en clarificar la definición de derechos humanos y los principios que lo abordan al mismo, entre otros aspecto que permitieron la determinación de este tema investigativo.

\section{CONCLUSIONES}

- Luego de haber analizado e interpretado los resultados, se recogen los hallazgos más importantes, y sobre la base de ellos se formulan las siguientes conclusiones al objeto de dejar aclarada la esencia investigativa innovadora los DDHH de las mujeres:

- Los Derechos Humanos, se consideran como la herencia que se adquiere desde la concepción, son derechos que son fundamentales tanto para el hombre como para la mujer. Resaltando que el proceso de reconocimiento de los derechos humanos de las mujeres llega a la objetividad y de concientizar de que la mujer tiene que ser vista como un sujeto, una persona con derechos, civiles, económicos, políticos, basado en igualdad y equidad.
- También, es importante resaltar que la consideración del reconocimiento de los derechos humanos de las mujeres era para que las misma pudieran desenvolverse libremente en todos los ámbitos que la misma quisiera llegar, su reconocimiento está, lo que no hay es conciencia por parte de un gran número de hombre machista que quieren seguir controlando la libertad y la vida de ellas.

- Por tanto, la Perspectiva de género empleada como método en el proceso investigativo conlleva a que se genere corpus reflexivos de ideas innovadoras ajustada a la actualidad, donde se consideran de una forma sistemática y hermenéuticamente interpretativa que el conjunto de normas internacionales en materia de derechos humanos de las mujeres es en pro de prevenir, erradicar y sancionar cualquier acto o conducta que se ejecute en contra de las mujeres por su condición de género.

\section{RECOMENDACIONES}

- Desde el punto de vista general, se le recomienda a la administración de justicia tanto nacional como internacional, a la sociedad académica investigativa universitarias en todos sus niveles, Pregrado, Postgrado, Doctorado $\mathrm{u}$ otros tipos de estudio, que generen ideas reflexivas con perspectiva de género, enfocada en la innovación, en proporcionar y proyectar más ideas propias, conocimientos y sabiduría a objeto de hacer un aporte donde se visualice la esencia del investigador y el mismo contexto investigativo sirva como referencia a otros investigadores a futuro. 


\section{REFERENCIAS BIBLIOGRÁFICAS}

- Castan (2020) El Papel de la Mujer Romana en la Política del S. I A. C. ¿Influencia Política, Participación Indirecta o Influencia Social?, Revista Internacional de Derecho Romano, ISSN 1989-1970, Link: file://C:/Users/ USER/Downloads/Dialnet-ElPapelDeLaM ujerRomanaEnLaPoliticaDelSIACInfluen ci-7359529\%20(1).pdf M [Consulta:2021, Octubre 10]

- Fundación Ayuda en Acción (2020) Cifras $y$ datos de violencia de género en el mundo (2020), Link: https://ayudaenaccion.org/ ong/blog/mujer/violencia-genero-cifras/ [Consulta:2021, Octubre 10]

- OMS (2021) La violencia contra la mujer es omnipresente y devastadora: la sufren una de cada tres mujeres, Link: https://www.who. int/es/news/item/09-03-2021-devastatinglypervasive-1-in-3-women-globally-experienceviolence [Consulta:2021, Octubre 10]

- Camacho (2016) El concepto de Derechos Humanos. El Dilema del carácter de los Derechos Humanos, Revista de Ciencias Sociales (Cr), vol. II, núm. 152, 2016 Universidad de Costa Rica San José, Costa Rica, Disponible: https://www.redalyc.org/ pdf/153/15348419001.pdf [Consulta:2021, Octubre 12]

- Mertus, Butegwa, Thomas y Schuler (2000) Derechos humanos de las mujeres: paso a paso. Guía para el uso del derecho internacional de los derechos humanos y de los mecanismos para defender los derechos humanos de las mujeres
/ Editado por el Instituto Interamericano de Derechos Humanos. - San José, C.R.: Instituto Interamericano de Derechos Humanos, Women Law \& Development International, Human Rights Watch Women's Rights Project, 2000. 260 pp.; 25.5 x 18 cms. (reimpresión).

- CNDH (2021) ¿Qué son los derechos humanos?, México, Disponible en: https:// www.cndh.org.mx/derechos-humanos/queson-los-derechos-humanos [Consulta:2021, Octubre 12]

- Fernandez (2021) La Victima Femicida una perspectiva desde la Autopsia Psicológica en la Criminología Forense en Venezuela, Universidad Yacambú, Barquisimeto, Venezuela, Disponible en: http://biblioteca. uny.edu.ve:8081/bonline/TG/TGM MCP 217.pdf [Consulta:2021, Octubre 12]

- Hurtado, J., (2004). Metodología de la investigación holística. Caracas: IUTP. Sypal.

- Martínez, M. (2001). Comportamiento humano. Nuevos métodos de investigación. (3era. Ed) México: Trillas

- Martínez, M. (2004). Ciencia y arte en la metodología cualitativa. México: Trillas.

- Morse, J (2003). Enfoques de la Triangulación Metodológica Cualitativa y Cuantitativa. México: Prentice Hall.

- Rodríguez, Gil y García (2001). Metodología de la Investigación Cualitativa. Málaga: Aljibe. 
- Rubio, J (2005). Positivismo, Hermenéutica y Teoría crítica en las Ciencias Sociales. Barcelona: Humanitas.

- Convención Interamericana para Prevenir, Sancionar y Erradicar la Violencia Contra la Mujer. Convención de Belém do Pará, OEA, (1994).

- Convención Sobre la Eliminación de Todas las Formas de Discriminación Contra la Mujer (1979).

- Declaración Universal de los Derechos Humanos (1948) 
